\title{
Investigación cualitativa y Etnografía. Perspectivas y desafíos: el caso del fenómeno religioso
}

\author{
Ado Amegeiras
}

\section{Resumen}

El trabajo que presentamos es el resultado de las reflexiones realizadas en el marco de nuestra práctica de investigación con metodologías cualitativas y especialmente con etnografía. Se trata de plantear algunas interrogantes y compartir apreciaciones vinculadas con la necesidad de enfrentar problemas de carácter ontológico como epistemológico, que nos conducen inevitablemente a resolver interrogantes metodológicas. Una situación que se presenta en el conocimiento de los fenómenos sociales en general y de los fenómenos religiosos en particular. En esta presentación, además de considerar los vínculos entre la investigación cualitativa y la etnografía, nos detendremos especialmente en el abordaje etnográfico del fenómeno religioso, considerando el caso de una peregrinación religiosa realizada por migrantes radicados en sectores populares del Gran Buenos Aires, en el cual es posible considerar los alcances y potencialidades de las metodologías propuesta.

Palabras claves: Investigación cualitativa / reflexión epistemológica / abordaje etnográfico/ / fenómeno religioso/

\section{Qualitative research and Ethnography. Perspectives and challenges: the case of the religious phenomenon}

\begin{abstract}
The present work is the result of the reflections that emerged in our researching practice with qualitative methods and especially with ethnography. We try to come up with several questions and share assessments related to the need of facing up to ontological and epistemological problems which take us inevitably to answer methodological questions. A situation that incurs in the social phenomenon knowledge in general and in the religious phenomenon in particular. On this exposition we consider not only the relation between the qualitative research and the ethnography, but also we particularly refer to the ethnographic approach of the religious phenomenon. In this sense, we will study in
\end{abstract}

* Profesor de la Universidad Nacional General Sarmiento de Buenos Aires. 
greater detail about the case of a religious pilgrimage of migrants who live in popular areas of the "Gran Buenos Aires" in order to consider the scopes and the possibilities of the proposed methodology.

Key words: qualitative research - epistemological reflection-ethnography-religious phenomenon

\section{Pesquisa qualitativa e etnografia. Perspectivas e desafios: o caso do fenômeno religioso}

\section{Resumo}

O trabalho que apresentamos é fruto das reflexões feitas no marco de nossa prática de pesquisa com metodologias qualitativas e principalmente com a etnografia. Seu objetivo é levantar algumas questões e compartilhar opiniões relacionadas à necessidade de enfrentar problemas ontológicos e epistemológicos que inevitavelmente nos levam a resolver questões metodológicas. Uma situação que surge no conhecimento dos fenômenos sociais em geral e, particularmente, dos fenômenos religiosos. Nesta apresentação, além de abordar os vínculos entre pesquisa qualitativa e etnografia, nos deteremos especialmente na abordagem etnográfica do fenômeno religioso, levando em consideração o caso de uma peregrinação religiosa feita por migrantes que vivem em áreas populares da Grande Buenos Aires, onde é possível considerar alcances e potencialidades da metodologia proposta.

Palavras-chave: Pesquisa qualitativa; reflexão epistemológica; abordagem etnográfica; fenômeno religioso

La investigación social cualitativa se encuentra en los últimos años ante nuevos desafíos relacionados tanto con la complejidad de la sociedad como con los reposicionamientos epistemológicos y metodológicos de la ciencia social. Una investigación social que se despliega en contextos en los que mujeres y hombres, niños y ancianos despliegan su existencia y en el cual, una gran mayoría, lo hacen en condiciones de vida precarias e injustas, atravesando procesos de exclusión y marginación social. Sectores cuyas voces no son escuchadas o sufren descalificación y encubrimientos en sus demandas e intereses. Una realidad que conmueve, que demanda respuestas y que nos interpela permanentemente a los investigadores sociales en un contexto caracterizado por una globalización marcadamente asimétrica y con profundas transformaciones científicas y tecnológicas. Esto implica abordar las responsabilidades y el compromiso ético de la investigación y especialmente resolver los desafíos epistemológicos vinculados con la forma y el modo en que construimos el conocimiento en la ciencia social, como con la perspectiva relacionada con el "desde dónde" y "para qué" lo estamos haciendo. De allí entonces, el surgimiento de numerosas preguntas; ¿Cómo fundamentamos 
nuestros conocimientos? ¿En qué se sustentan nuestras interpretaciones? ¿Cómo se expresa la voz de los sujetos que investigamos? ¿Qué lugar ocupan sus apreciaciones e interpretaciones de la realidad? Finalmente, la pregunta que ha acompañado muchas veces las dudas de los investigadores sociales ¿Quiénes somos nosotros para hablar sobre ellos y por ellos? Un tipo de planteamiento que atraviesa diversas áreas disciplinarias y que ha dado lugar a importantes debates que han enriquecido la ciencia social pero que implican fuertes controversias y perspectivas de abordaje.

Especialmente en los últimos tiempos observamos cómo han resurgido de la mano de los cambios acaecidos y la llamada crisis de la modernidad, nuevos cuestionamientos de los paradigmas tradicionales acompañados por la paulatina emergencia de nuevos modelos. Una situación en donde se agudiza la gravitación de los posicionamientos que reclaman dar visibilidad a ese "desde dónde" los investigadores llevan a cabo sus aproximaciones a la realidad social y con cual finalidad lo hacen. Una instancia en donde el "para qué" de la investigación no puede soslayar compromisos éticos ni tampoco posicionamientos sociales tensionados y desvirtuados, generalmente bajo los argumentos de la "neutralidad valorativa", los requerimientos de la imprescindible "objetividad" o los supuestos en general de la investigación científica hegemónica. Nos encontramos de esta manera, con una diversidad de interrogantes en las cuales se ven interpelados tanto nuestros presupuestos de investigación como la relación con los sujetos que investigamos. Preguntas relacionadas con las formas tradicionales del conocer como con los desafíos relacionados con la validez o el surgimiento de nuevas metodologías, que escapan a las formulaciones legitimadas por el mundo académico. Problemas de carácter ontológico como epistemológico y que nos conducen inevitablemente a resolver nuestras interrogantes metodológicas. Posicionamientos y perspectivas que, en última instancia, colocan al investigador en una situación de tensión con respecto al tipo de ciencia social que ha marcado hasta el momento nuestra práctica profesional. Enfoques que ponen en duda nuestros marcos interpretativos y nuestras categorías de análisis junto a nuestras pretensiones de validez, sustentadas en gran parte, en esquemas positivistas en el marco de un paradigma modernizante. Como señala I. Vasilachis:

...la que se propone y avecina es otra ciencia, no ajena al requisito de la intersubjetividad, pero con principios, criterios y parámetros amplios, flexibles, 
sensibles a las particularidades y diferencias individuales y locales, con epistemologías múltiples, con metodologías nuevas y renovadas"(Vasilachis, $2012 ; 23)$

Es evidente que los desafíos son grandes y complejos, pero debemos aclarar que, si bien no es nuestra intención detenernos y profundizar en la búsqueda de respuestas a los núcleos centrales de esta problemática, si nos interesa explicitar algunos aspectos que consideramos imprescindibles de tener en cuenta.Se trata de aspectos vinculados con nuestros posicionamientos, como también de alentar una mirada crítica pero constructiva sobre estos temas. Advertir una vez más, sobre un momento en que los investigadores nos sentimos muchas veces como si estuviéramos parados en la cubierta del Titanic, a la vista de los primeros témpanos de hielo y urgidos por la necesidad de definir posicionamientos y tomar decisiones.

De ahí nuestra apreciación respecto a la necesidad de producir un profundo reposicionamiento epistemológico y metodológico en la ciencia social, como en sustentar la potencialidad de la investigación cualitativa en general y de la etnografía en particular, para abordar las complejidades de la actual realidad social. Una investigación cualitativa que constituye a nuestro entender, una forma de conocer y desarrollar el trabajo de investigación enmarcada en un nuevo planteamiento paradigmático y epistemológico. Es en dicho marco que la estrategia etnográfica, constituyendo una más de las estrategias cualitativas, emerge a nuestro entender con una especial potencialidad para el trabajo y el posicionamiento del investigador en el campo.

Una estrategia cualitativa que presenta una gran capacidad hermenéutica y facilitadora de prácticas cognitivas. Una apreciación estrechamente relacionada con nuestra convicción respecto a considerar que son los sujetos, los protagonistas de la vida social en sus múltiples posicionamientos y actividades. Sujetos ubicados espacio temporalmente, hacedores de relaciones, enmarcados en tramas conflictivas, pujas de intereses y predominios hegemónicos, que construyen, reconstruyen y asumen determinadas modalidades de vivir y de expresar su vida en distintas situaciones. Sujetos que no solo poseen la capacidad de conocer sino también de interpretar y de manifestarse en prácticas y relatos cuyas argumentaciones y densidad teórica, explicita la presencia de otras lógicas y racionalidades no disociadas de las hegemónicas pero diferentes e incluso alternativas. Sujetos interactuando con el sujeto investigador en un proceso social no exento de encuentros y 
desencuentros, de desigualdades y asimetrías frente al desafío de construir conocimiento.

\section{Primera aproximación: Algunos planteamientos, muchos cuestionamientos}

Nos estamos refiriendo a una situación que consideramos predominante y que se expresa fundamentalmente en poseer, más que la pertinencia de algunos planteamientos la certeza de muchos interrogantes que no son solo resultados de nuestras preocupaciones teóricas sino fundamentalmente de nuestra práctica de investigación. En ese sentido, nuestra reflexión asume una propuesta vinculada con la necesidad de que los investigadores reflexionemos sobre nuestra propia práctica y sobre la manera en que construimos conocimiento. Un planteamiento que desde el un primer momento sustenta la necesaria articulación entre los supuestos paradigmáticos, los presupuestos teóricos y las propuestas metodológicas. Una instancia que nos invita imprescindiblemente, a reflexionar en torno a las formas y los modos en que dichos supuestos y posicionamientos gravitan y singularizan nuestras estrategias de investigación.

Una perspectiva que nos enfrenta entonces, en primer lugar, con la llamada crisis de paradigmas y que se despliega a través de los cuestionamientos de perspectivas teóricas y metodológicas, de las disputas, como también de los reposicionamientos epistemológicos que derivan en la emergencia de nuevos paradigmas y el reconocimiento de la pluralidad de voces y saberes. (Denzin N. Lincoln Y, 2012:37). Una situación que se corresponde con una crisis generalizada de creencias y cosmovisiones que no solo afectan a los actores sociales en general sino también a los investigadores sociales y que se explicita entre otros aspectos, en las limitaciones de los marcos interpretativos con los cuales dichos investigadores tratan de abordar y comprender la realidad. (J.Varela/ Alvarez-Uría F,1997:8). Una situación que implica en el trabajo, la apelación a distintas conceptualizaciones y perspectivas teóricas y metodológicas enmarcadas en propuestas paradigmáticas. De ahí que resulta necesario tener en cuenta que el paradigma, en tanto constituye un "sistema básico de creencias o cosmovisiones que guían al investigador" tiene consecuencias ontológicas y epistemológicas. Guba. E, /Lincoln. Y (1994:105) y que resulta imprescindible analizar las mismas para lograr una comprensión integral de la problemática en cuestión. Una afirmación que abre un camino en el 
cual cobra visibilidad, según dichos autores, no solo el tipo de ontología en juego y sus apreciaciones respecto a un realismo de características ingenuas, críticas o históricas sino también perspectivas de consideración de la realidad que presentan el carácter en que la misma pueda ser asumida. Un tipo de asunción que puede adquirir una perspectiva dualista, objetivista o subjetivista y que deriva de hecho en propuestas metodológicas que asumen según el paradigma un carácter experimental, manipulativo, dialéctico u hermenéutico (E. Guba Y-Lincoln-1994:106). Una mirada de estos autores profundizadas en otros trabajos en los cuales refiriéndose en particular a las controversias paradigmáticas como a las contradicciones y confluencias emergentes, avanzan en reconocer las dificultades para la concreción de un paradigma de carácter único o un discurso común.

nos encontramos en el umbral de una historia marcada por la multivocalidad, los significados refutados, las controversias paradigmáticas y las nuevas formas textuales. A cierta distancia de esta senda conjetural, cuando se escriba su historia encontraremos que esta ha sido una era de emancipación; emancipación de aquello que Hanna Arendt denomina "la coerción de la verdad" emancipación del hecho de oír solo las voces de Europa occidental, emancipación de generaciones de silencio y emancipación de ver el mundo de un solo color. (Guba y Lincoln,2012:70)

Una apreciación sobre los paradigmas en la ciencia social que ha transitado por un extenso recorrido a lo largo del cual se han manifestado los enfrentamientos y las pujas, las instancias de posicionamientos fundamentalistas como los posicionamientos de coexistencia paradigmática, el predominio de paradigmas hegemónicos como la reacción de paradigmas alternativos.(Flick.U, 2004) Una situación en que el paradigma positivista ha ejercido y en gran parte sigue ejerciendo, un predominio fundamental estableciendo o pretendiendo hacerlo, no solo los supuestos y condiciones que marcan la cientificidad o no de determinados abordajes sino a su vez los criterios de rigurosidad y validez que deberían acompañar los mismos. Un paradigma que se constituyó en el hito de referencia de lo que supuestamente debía constituir la forma legitimada de conocer frente a otras, que no habiendo sido ser totalmente desplazados, sin embargo, fueron descalificadas por su falta de rigurosidad, seudo cientificidad o sencillamente en muchos casos por una supuesta marcada irracionalidad. Pero un paradigma cuyas implicancias políticas y sociales convalidaron formas de conocer e intervenir 
sobre la realidad con múltiples implicancias políticas, sociales y culturales. Posicionamientos frente a los cuales los enfoques provenientes del marxismo generaron un cuestionamiento fundamental, consolidado en sus apreciaciones por los aportes posteriores de la teoría crítica en forma análoga a la manera en que los enfoques post positivistas intentaron mitigar las ortodoxias positivistas. De ahí la necesidad de un nuevo paradigma, claramente en construcción, receptor de críticas y reposicionamientos, pero con una dinámica generada por distintas vertientes que confluyeron creativamente en dicha propuesta (Vasilachis de Gialdino I,1992). Un nuevo paradigma que demanda la necesidad de considerar la capacidad de los sujetos de significar su vida, de reconocer tanto su capacidad y derechos como seres humanos,como de decir su voz. Un nuevo paradigma surgido en el marco de posicionamientos hermenéuticos, fenomenológicos y constructivistas que abrieron las puertas a otros enfoques que se suman o confrontan con los anteriores como el paradigma de la participación, el paradigma de la cooperación, el paradigma feminista o el de los estudios culturales, entre otros (N. Denzin, Y. Lincoln, 2012). Una instancia que pone sobre la mesa relatos contrapuestos, nuevas miradas y enfoques que convocan a una apertura de pensamiento, que no solo incorpora el lenguaje de Wallerstein I (1996), en términos de la necesidad de "abrir la ciencia", sino que avanza decididamente en pensar creativamente en el cuestionamiento de dicha ciencia desde nuestro posicionamiento existencial y espacial. Un posicionamiento receptivo y expresivo frente a la magnitud de la exclusión social como ante los reclamos de los pueblos originarios, la demanda de las mujeres, la exclusión de migrantes y desplazados como en general de todas aquellas minorías marginadas.

Es que en la actualidad más allá de la emergencia de nuevos paradigmas presenciamos la existencia de un profundo cuestionamiento que supone el despliegue de una mirada que coloca el saber y los desafíos de la construcción del conocimiento en el marco de un proceso mayor de enorme complejidad. Un proceso en el cual se dirimen las relaciones del poder y se visibilizan las asimetrías existentes. Una perspectiva que se enmarca en un cuestionamiento profundo a una modernidad capitalista que ha colocado en el llamado "Paradigma dominante" (Boaventura De Souza Santos, 2009:31) el centro de sus críticas en cuanto el mismo en su pretensión de universalidad y homogeneización ha descalificado y marginado cualquier otro posicionamiento filosófico, teórico y metodológico de conocimiento. Un paradigma que al 
decir del mismo autor ha entrado sin embargo, en una profunda crisis como ocurre en realidad con el modelo de racionalidad científica que lo caracteriza. Una racionalidad que no solo se expandió sustentando la primacía de la racionalidad moderna sino que, como el mismo enfatiza, se constituye en un tipo de racionalidad científica generadora de "un modelo totalitario en la medida en que niega el carácter racional a todas las formas de conocimiento que no se pautan por sus principios epistemológicos y por sus reglas metodológicas. "(Boaventura De Souza Santos, 2009: 21)

De ahí la necesidad de la emergencia de un nuevo paradigma que lejos de auto arrogarse predominios absolutos de racionalidad sea capaz de reconocer otras racionalidades y otros logos .(Boaventura. De Souza Santos, 2009:31). Es aquí en donde la emergencia de nuevos paradigmas nos conduce a reposicionamientos epistemológicos imprescindibles de considerar al reflexionar sobre los procesos de construcción del conocimiento. El mismo Boaventura da un paso en ese sentido al proponer la necesidad de plantearse avanzar hacia la construcción de una "epistemología de Sur";

...la búsqueda de conocimientos y de criterios de validez del conocimiento que otorguen visibilidad y credibilidad a las practicas cognitivas de las clases, los pueblos y los grupos sociales que han sido históricamente victimizados, explotados y oprimidos por el capitalismo y el colonialismo global. (Boaventura De Souza Santos, 2009:12)

Reposicionamientos epistemológicos que han surgido de la mano de procesos de descolonización como de la emergencia de actores sociales que reclaman no solo expresar su propia voz sino también el reconocimiento de su forma de ver y pensar la realidad en la que están insertos. (Bidaseca K, 2010:117) Una perspectiva que resultó fecundada por presupuestos que surgidos ya desde principios de siglo en Latinoamérica con autores como Mariátegui, D Ribeiro, E-.Dussel, F .Ortiz, Fernández Retamar o R. Kusch entre otros, advirtieron tempranamente sobre la necesidad de cuestionar el "discurso hegemónico" (Castro Gomez,1998). Si bien, en gran parte, varios de ellos habían sido fuertemente criticados o deslegitimados por una academia hegemonizada por modelos eurocéntricos y modernizadores. De ahí, también el fuerte cuestionamiento que viabiliza en su reflexión E. Lander al señalar la necesidad de "buscar alternativas" a este mundo moderno "excluyente y desigual" que universaliza un modelo de sociedad "capitalista liberal." 
...esto requiere el cuestionamiento de las pretensiones de objetividad y neutralidad de los principales instrumentos de naturalización y legitimación de este orden social: el conjunto de saberes que conocemos globalmente como ciencias sociales.(E. Lander, 2000: 4)

Distintos enfoques que plantean con claridad la necesidad de una nueva epistemología y que encuentran en la emergencia de nuevas teorías y planteos hitos referenciales indispensables para legitimar nuevas formas de conocer y abordar la realidad social. Una perspectiva vinculada con las teorías poscoloniales y subalternas como los cuestionamientos de la filosofía latinoamericana y la teología de la Liberación. Autores como E. Said en Medio Oriente, R. Guha, H. Bhabha o Gayatri Spivak en la India, MBembé y Y Mudimbe, en África o A. Quijano, E. Saer y Castro Gómez en Latinoamérica entre muchos otros, son una muestra enormemente significativa de lo expuesto. Posicionamientos en donde el llamado "encubrimiento de América" de E. Lander (2000) o la idea de "Colonialidad del poder "de A. Quijano (2000) posibilitan y a la vez convocan a reposicionamientos imprescindibles en nuestros enfoques epistemológicos. Como señalan acertadamente R. Briceño Leon y H. Sonntag (1999:103) "la ciencia social tiene una cuenta pendiente con la singularidad de América Latina."

Al realizar este recorrido no podemos dejar de destacar y profundizar, algunos aspectos presentes en el pensamiento de R. Kusch, por la relevancia del mismo, en el esfuerzo por realizar reposicionamientos epistemológicos imprescindibles en nuestra práctica de investigación. Es que el autor sostiene que las dificultades para producir un conocimiento adecuado requiere una inmersión en las culturas de los pueblos de América, de manera de descubrir a partir del pensamiento indígena, los saberes arraigados territorialmente y los pliegues de lo popular, un pensar "propio". Es en el marco de sus búsquedas no sustentadas en los tratados filosóficos ni en los ricos legados de los pensadores occidentales consagrados, sino en el contacto directo, las largas conversaciones con indígenas y campesinos, sus recorridos por el altiplano y sus caminatas por el Cuzco que encuentra las claves del pensar propio de su "América profunda", que expresa con claridad en sus obras. (Kusch R,1975)

Es desde allí desde donde plantea frente a las tensiones del ser y las angustias del existir la relevancia del "estar" que no se reduce a una mera instalación, sino que implica el despliegue de un "hábitat" capaz de dotar de sentido al esfuerzo permanente por ponderar la vida sobre la muerte. El tema 
del "estar" aparece como el resultado de una mirada diferencial respecto a la apreciación del "ser" pero implica a su vez una síntesis que se expresa en el "estar siendo" que no excluye las contradicciones, sino que las incorpora en un posicionamiento pleno de sentidos. Está en juego una manera de encarar la vida en que el futuro se define en el aquí y ahora de la lucha por una existencia profundamente amenazada por un mundo desigual y excluyente. De allí que el autor señala que

... el pueblo no vive su cultura como un simple entretenimiento sino como una forma de concretar en una fecha determinada, o en un ritual cualquiera el sentido en que intuidamente descansa su vida. (Kusch R , 1978: 19)

Se trata de una idea que pondera la cultura como un "domicilio existencial" que constituye un desafío fundamental para el investigador que se aproxima a la misma, pero que cuando lo hace, necesariamente se encuentra ante un "espacio intercultural" en donde se generan prácticas sociales y simbólicas no exentas de tensiones y conflictos. Es que se trata de un "pensamiento seminal" que caracteriza al pensamiento indígena y nutre las características de la cultura popular. Como señala C. Parker.

... Una cultura popular, mucho más simbólica dramática que intelectual. Con toda su sabiduría popular representa" otra lógica... mucho más empírico y simbólico a la vez, mucho más sapiencial y dialéctico que cartesiano y positivista.. (Parker C, 1993: 370)

Una propuesta que implica una nueva epistemología que nos conduce a plantearnos aspectos teóricos y metodológicos directamente relacionados con nuestras estrategias de investigación, Está en juego la forma y el modo en que enfrentamos el abordaje de la realidad social y muy especialmente el lugar que ocupan los sujetos con los que nos vinculamos en dicho proceso de investigación. Una situación en que los conflictos epistemológicos se traducen en un dominio generado en una larga tradición. Como señala Irene Vasilachis, (2003:25) en el despliegue de una metaepistemología en la que considera la complejidad y la tensión existente entre la denominada "epistemología del sujeto cognoscente" y la "epistemología del sujeto conocido". En el primer caso una epistemología caracterizada según la autora por una "ontología realista" que en última instancia no puede sustraerse a considerar a los sujetos como "observables" y externos a quien tiene la capacidad y la legitimidad cognoscente en el actual contexto. Una situación 
en la cual solo le corresponde al otro sujeto ser conocido. Un sujeto que a lo sumo espera que sea interpretado adecuadamente en cuanto se encuentra cercenado en manifestar abiertamente su capacidad de conocimiento y en última instancia la legitimidad y validez de su pensamiento. La autora insiste en este punto en la necesidad de otorgar visibilidad no solo a las formas objetivas sino a las subjetivas en donde se traslucen los sentimientos, los valores y las apreciaciones del sujeto, sustentando una nueva epistemología que denomina precisamente como del "sujeto conocido" que si bien se inserta en un paradigma interpretativo, constructivista o hermenéutico marca una diferencia central.

A diferencia de las principales orientaciones que inician y consolidan el paradigma interpretativo, no se trata ya "ni de entender la acción social interpretándola"(Weber, 1944:4) ni de "comprender sus motivos para y porqué"(Shultz, 1972:58) ni de "hacer explícita la significación dada" (Habermas, 1985:41) por los actores sociales a su acción en el proceso de conocimiento; de lo que se trata es de considerar el resultado del proceso de conocimiento como una construcción cooperativa en que los sujetos esencialmente iguales realizan aportes diferentes. (Vasilachis I, 2003:30)

Un posicionamiento epistemológico que exige reconocer, tanto la capacidad de conocer, como la capacidad de interpretar de los sujetos con los cuales el investigador interactúa. Un posicionamiento epistemológico que se relaciona inevitablemente con nuestras propuestas de investigación y que nos conduce a rever iniciativas que en muchas oportunidades insisten con conceptualizaciones, categorías y estereotipos que lejos de aportar al conocimiento terminan sumando nuevas "estigmatizaciones" al sujeto en cuestión.

Apreciaciones largamente fundamentadas por Vasilachis quien enfatiza la necesidad de "romper con todo conocimiento que suponga violencia de unos sobre otros..." en tanto las mismas suponen desconocer la capacidad de conocimiento y reflexión de los sujetos. Una perspectiva cualitativa indispensable en este contexto para comprender la densidad de lo humano en la trama compleja y conflictiva del mundo de la vida.

Resulta interesante asimismo tener en cuenta las apreciaciones $\mathrm{N}$. Denzin e Y Lincoln (2011) quienes aún en otra perspectiva consideran que si bien las distintas metodologías, ya sean cuantitativas como cualitativas ponen en juego siempre sus apreciaciones sobre el otro, dichas apreciaciones son distintas. De ahí que pasan a destacar especialmente las potencialidades de 
la investigación cualitativa en cuanto a constituirse en una estrategia que no solo reconoce múltiples formas de conocimiento y modalidades culturales, sino que constituyen "una actividad situada..." por lo cual los investigadores cualitativos "tratan de entender o interpretar los fenómenos en función de los significados que las personas les dan”. (N. Denzin, Y. Lincoln, 2011: 48). Un énfasis en la "situacionalidad" que consideramos importante no solo en cuanto implica otorgar relevancia a los contextos de interpretación, sino porque dicha interpretación coincide de alguna manera con lo planteado por R. kusch . Es que para este autor se requiere imprescindiblemente del desarrollo de un "pensamiento situado", de un pensar "culturalmente arraigado" (Mareque E,1989: 53) pero en el que el contexto sobre la base del soporte espacial y social alude no solamente a un contexto territorial sino muy especialmente a un contexto existencial. Así, lo epistemológico en esta nueva perspectiva requiere explicitarse en una metodología que asuma como presupuesto fundamental, la necesidad de comprensión de los sujetos protagonistas de la acción social, respetando su identidad esencial como su diferencia existencial (Vasilachis, 2003:27). Una metodología de carácter cualitativo especialmente adecuada en un momento en que un desafío fundamental de investigación pasa por escuchar a los que nunca han sido escuchados, por legitimar su capacidad de conocer e interpretar y decir su palabra Un planteo imprescindible frente a la hegemonía de otros discursos, relatos y propuestas que bajo la etiqueta de la universalidad y la cientificidad terminan imponiendo su perspectiva del mundo y de la vida.

\section{Segunda aproximación: Desde la investigación cualitativa al abordaje etnográfico}

De esta manera, nos interesa profundizar las posibilidades de la investigación cualitativa, a partir de esta apreciación que estamos sustentando. Métodos cualitativos sustentados en un paradigma hermenéutico que implica básicamente el requisito de la comprensión y el ejercicio de la interpretación, constituyéndose en un recurso adecuado para abordar los enormes desafíos que requiere un reposicionamiento epistemológico. También en esa dirección es interesante el posicionamiento de Flick (1982:2) para quien la investigación cualitativa constituye un instrumento importante para enfrentar el desafío de comprensión de un mundo inmerso en importantes transformaciones como el actual. Un contexto en donde se agudiza la diversidad de perspectivas y los cruces culturales. Una metodología de investigación cualitativa que 
por su capacidad de apertura y su énfasis en conocer el punto de vista de los otros y la manera en que significan al mundo implica una estrategia relevante de conocimiento de la realidad. Una metodología flexible, que requiere no solo la presencia de los investigadores en el contexto de los actores sino fundamentalmente una activa comunicación con los mismos que permita la comprensión de los sentidos presentes en la acción social. Una metodología dinámica inserta en un paradigma interpretativo que pondera la búsqueda de la comprensión y el descubrimiento por sobre la explicación y la verificación en cuanto es a partir de dicho contexto de descubrimiento que se plantea construir teoría. Al respecto es importante recordar que la investigación cualitativa en cuanto tal supone una diversidad de perspectivas de investigación y estrategias metodológicas y que la definición de la misma será acorde en cada caso a los distintos enfoques y situaciones. Sin embargo, es claro que podemos hablar en términos generales por el interés en un abordaje que intenta comprender, interpretando los significados que las personas otorgan a sus comportamientos como los sentidos presentes en la trama social y en la urdimbre cultural( Maxwell,2004; Silverman, 2005). Una comprensión y un esfuerzo interpretativo que necesariamente implica la consideración de los contextos y los procesos en que se despliegan las distintas tramas y matrices culturales. Perspectiva que entraña considerar la relevancia del lenguaje, de las experiencias y vivencias de los sujetos, de la fecundad de sus saberes y de las diversas prácticas sociales y simbólicas que nutren la habitualidad constitutiva de la vida cotidiana.

Es aquí en donde nos interesa revalorizar la idea de Vasilachis (2006:59) respecto a las posibilidades que brinda la epistemología del sujeto conocido en relación a transformar nuestros presupuestos ontológicos. Un planteo acorde con lo cual el investigador tiene la posibilidad de abandonar posicionamientos asimétricos con los sujetos que conoce como avanzar en un horizonte de sentido que los identifica como seres humanos. Requerimientos que suponen el esfuerzo de los investigadores por agudizar su capacidad de escuchar y de aprender de los otros con los que se relaciona e interactúa. Un aprendizaje no solo explícito en relación a la trama de sentidos en que los actores están insertos en un contexto determinado sino también en la perspectiva de un investigador que hace de su práctica profesional una experiencia que transforma su posicionamiento vital. De ahí que, señala Vasilachis "esta manera de investigar es considerada como una forma de pensar más que como una colección de estrategias técnicas".(2006:27). La investigación 
cualitativa desde esta perspectiva recupera una tradición de la ciencia social reivindicada por Wright Mills respecto a considerar que, "hacer investigación social es la práctica de un oficio". Una apreciación que en cuanto tal se constituye en una verdadera artesanía intelectual, en cuanto demanda una disposición de trabajo que desde la valorización de la experiencia vivencial y la subjetividad del investigador interactúa y se vincula con otros en lo común que los identifica y en las marcadas diferencias que los distinguen. Un trabajo, insistía Mills, en que era necesario “aprender a usar la experiencia de la vida...examinándola e interpretándola sin cesar “(C.Wright Mills,1961:207)

Una investigación cualitativa de carácter multi-metodológico que plantea distintas estrategias vinculadas como señala Creswell (1998:15) con tradiciones metodológicas. Nos estamos refiriendo a la teoría fundamentada en los datos, el estudio de casos, la biografía y la etnografía sobre la que nos interesa detenernos en particular en cuanto, más allá de su relevancia en la antropología, ha sido revalorizada en los últimos tiempos como una estrategia de enormes posibilidades en el trabajo de investigación social cualitativa en general. Es que, como señala J. Spradley (1979:3) la etnografía más que estudiar a las personas significa "aprender de las personas" en un esfuerzo de comprensión e interpretación del significado que las acciones y acontecimientos tiene para los mismos. Como señalábamos en un trabajo anterior

Toda la tarea de investigación para el etnógrafo es un ejercicio de sentido tanto en relación a los "otros" como respecto a él mismo, ambos sujetos productores de sentido, comprometidos en un tipo de práctica comunicativa que hace posible un horizonte de comprensión común.. (Ameigeiras, 2006:119)

La noción de cercanía en la que el investigador desarrolla su tarea en el campo agudiza el esfuerzo interpretativo del otro en un esfuerzo de comprensión de su identidad. Una tarea que requiere no caer en estereotipaciones preconcebidas ni en adjudicaciones que resultan más de los propios marcos interpretativos del investigador que del arduo proceso de diálogo y construcción cooperativa del conocimiento. Si de replanteo epistemológico se trata, el mismo supone una tarea de desciframiento en la cual "ninguno tiene la capacidad de establecer un monopolio de la interpretación "(W Iser, 2005:26) acorde con un proceso que se abre y en el que está en juego la "traducción” y el carácter transformativo que posee la misma. Una situación que requiere el despliegue de negociaciones de sentido 
que implican asociaciones y consensos resultado directo del contacto y la proximidad con el otro.

Un esfuerzo en que el investigador se encuentra expuesto a una interpelación que lo deconstruye en la certeza de sus marcos interpretativos para avanzar en un proceso que, en cuanto legitima la capacidad de conocer e interpretar de los otros lo inserta en un campo intercultural de replanteos y transformaciones. Una situación en que el trabajo permanente de ejercicio de la reflexividad del etnógrafo se encuentra expuesto a múltiples transformaciones en que se modifican apreciaciones y habilitan nuevas miradas y perspectivas. Una instancia sustentada en el requisito básico de la investigación etnográfica demandante de "un aprendizaje", que como señala con profundidad R. Guber implica considerar que el investigador "aprende a mirar y a escuchar del modo en que lo hacen sus interlocutores o sujetos de estudio" (R.Guber, 2013: 326). Es que el trabajo de campo en la etnografía "sigue siendo un método inusualmente sensitivo, requiere un arduo aprendizaje del lenguaje y a menudo un desarreglo de las de las expectativas personales y culturales" (J. Clifford, 1992:143).

Estamos aludiendo a una reflexividad que se despliega en el trabajo de campo como ámbito fundamental en donde se producen las relaciones sociales y se construyen las interpretaciones. Un trabajo de campo en donde se nutre la experiencia del investigador y se producen nuevas vivencias (Galindo Cáceres, 1998:71). Una apreciación que encuentra en las palabras de E. Rockwell una enorme significación,

...la experiencia de campo debe transformar al etnógrafo. Sino se vive una transformación profunda de los marcos de percepción, de interpretación y de comprensión de la localidad en que se realizó el estudio, el largo trabajo de cambio y de análisis cualitativo no tiene sentido. (E.Rockwell,2009:185)

Una estrategia etnográfica que atravesó tempranamente cuestionamientos epistemológicos y que avanzó tanto sobre la denominada "autoridad etnográfica" como sobre la" relevancia textual" de la etnografía transitando entre experiencias y la búsqueda de distintos lenguajes etnográficos. Ya no se trata solo de "interpretar al otro" sino "de interpretar con los otros" lo cual implica un giro fundamental tanto de la antropología reflexiva como de la sociología interpretativista. En este punto resulta interesante lo señalado por P. Wright al definir la etnografía como una 
"desplazamiento ontológico lo que implica que al ser el etnógrafo su propio instrumento de registro y análisis de datos, una marca de fábrica peculiar entre las Ciencias Sociales (Levi-strauss,1976;36) el se desplaza ontológicamente a través del mundo"(P. Wright 2008 ;231).

Una perspectiva del autor que de alguna manera se articula con el énfasis de Vasilachis en un reposicionamiento ontológico del investigador pero que también está presente en el pensamiento de Kusch. Es que para este autor lo que prima no es una apreciación existencialista de un "ser arrojado al mundo" sino una "instalación" en un lugar y en un "suelo" en donde la cultura genera una trama de sentidos. (R.Kusch,1978:94) Se trata indudablemente de una experiencia del etnógrafo en el campo en el que no solo se modifican categorías sociales sino que se lleva a cabo "un arduo proceso de descolonizar el conocimiento en diálogo con otros”. E. Rockwell (2009; 186).

\section{Tercera aproximación: El abordaje etnográfico del fenómeno religioso}

Hemos avanzado hasta aquí en generar una reflexión sobre las características de la investigación cualitativa y la singularidad de la estrategia de investigación etnográfica, nos interesa ahora detenernos en el abordaje etnográfico del fenómeno religioso en particular. Consideraremos de esta manera las singularidades de este fenómeno y la relevancia de la etnografía para el conocimiento de lo sagrado que se manifiesta en Latinoamérica. Aspectos que agudizan el requerimiento de nuevos posicionamientos epistemológicos y paradigmáticos que permitan un abordaje adecuado y un conocimiento profundo de dichos fenómenos (J. Vigil, 2014:162). Una perspectiva en la cual la necesidad de aproximaciones hermenéuticas emerge como imprescindibles en contextos complejos de cruces culturales, superación de las fronteras religiosas y agotamiento de categorías y formas tradicionales de conocimiento. (P. Sanchis, 1998; Parker C, 2011; E. Rodriguez, 2014 y P. Barrera, 2014)

El nuevo paradigma interpretativo deberá centrarse mucho más en los espacios de transacción que en la configuración de los centros de definiciones sustantivas de cada sistema religioso. (C. Parker, 2011:37)

De esta manera las "fronteras móviles y porosas" entre sistemas como entre simbolismos religiosos requiere de nuevas perspectivas interpretativas 
pero también de propuestas metodológicas que reconociendo la capacidad cognitiva e interpretativa de los sujetos avance en un proceso existencial de comprensión de los fenómenos. Una situación que acrecienta la relevancia de la investigación cualitativa y de la etnografía en particular en cuanto la misma implica un posicionamiento existencial y experiencial en las relaciones con los otros sujetos en el territorio que despliegan su vida cotidiana.

De hecho estamos ante una estrategia de investigación a la que han apelado una gran mayoría de investigadores en sus investigaciones y que explicitan en sus trabajos las potencialidades de la etnografía para el abordaje del fenómeno religioso (C. Steil , 1996;C. Castella Vazquez,2015; Lewgoy B, 2004; P. Wright,1998;R.- de la Torre, 2012;S. Montenegro, 2012; N. Viotti; G. Ludueña, 2006;Algrani J, 2008; Ameigeiras, 2006) Es que en el abordaje etnográfico del fenómeno religioso es posible observar tanto el desarrollo de una minuciosa descripción de prácticas sociales y simbólicas como de ritualidades y comportamientos, pero fundamentalmente de un posicionamiento y un compromiso experiencial del investigador con el otro. Un sujeto con el cual interactúa que se constituye como sujeto dialógico y relacional en un espacio, un momento y un tiempo en el aquí y ahora de su existencia. Una perspectiva desde donde avanzar en la comprensión de los significados que los sujetos otorgan a sus creencias y comportamientos como de las configuraciones de sentido que se construyen en el marco de la trama socio cultural de la sociedad. Es interesante lo explicitado por P. Wright (1998:190) al respecto:

En tanto el antropólogo pueda aceptar ser sujeto hablante de un "mundo" que desea conocer, su tarea estaría dirigida a tratar de comprenderlo a través de la propia experiencia de su ser-en-el-mundo, desplazado ontológicamente por un paisaje contingente y fluido."

$\mathrm{El}$ autor insiste especialmente, coincidiendo con otros autores como Paden (1998) en la necesidad de tener en cuenta el lenguaje "propio" de la religión que no solo implica su "sintaxis" sino entender que se trata de "un conjunto total, vital" no reducible solo a creencias. Afirmaciones que consolidan una mirada que recupera el bagaje existencial, las actitudes vitales, las interacciones de los sujetos con aquello que consideran como sagrado y en relación con el cual organizan su cosmos personal y colectivo. Una mirada y un posicionamiento etnográfico en cuanto se trata de comportamientos, 
actitudes, gestos y miradas que requieren de una perspectiva especial del investigador en el campo.

Nuestra experiencia de campo nos ha posibilitado al respecto consolidar nuestra apreciación respecto a la necesidad de un posicionamiento que nos involucra básicamente como sujetos con otros sujetos tratando de comprender la complejidad en que se despliega la vida cotidiana. En nuestro trabajos sobre las fiestas populares religiosas, tanto en relación a migrantes de las provincias a los centros urbanos como también a los migrantes provenientes de países limítrofes,el abordaje etnográfico ha constituído una estrategias fundamental para la construcción de conocimiento.( Ameigeiras A,/1992/2002/2002b) De esta manera la apelación a la etnografía ha contribuído a la ampliación de nuestra comprensión de lo humano enfrentado a los grandes desafíos de la existencia,el sentido de la vida y de la muerte, la salud y el dolor, el goce y la tristeza. Situaciones y experiencias de los sujetos en los cuales se hace presente la experiencia de lo sagrado.

Si bien han sido numerosos los abordajes que realizamos vinculados con las creencias y las prácticas de los migrantes como de los fenómenos religiosos en general nos interesa especialmente hacer una alusión a una situación de campo en particular. Nos referimos a un viaje realizado con un grupo de migrantes santiagueños que iban a la fiesta del Cristo de Mailín en Santiago del Estero. Se trata de una fiesta originaria del siglo XVIII realizada a partir del hallazgo de una cruz en el tronco de un árbol (algarrobo árbol sagrado para los indígenas de la zona) y que todos los años se realiza originariamente en el pueblo de Mailín y a la misma vez en el gran Bs. As. en el barrio Guadalupe de la localidad de villa de Mayo. Muchos devotos que residen en el conurbano y que son generalmente asiduos concurrentes a la fiesta de Villa de Mayo, si pueden, se trasladan a Santiago del estero a pasar la fiesta, a $1200 \mathrm{Km}$ de Bs. As., para estar en su tierra y en el lugar de origen de la devoción. Con el motivo de participar de ese tipo de evento me sumé a un viaje que se promocionaba a Santiago del Estero por las radios barriales de manera informal para los que querían participar de la fiesta. La mayoría de los que viajaban eran migrantes santiagueños, que regresaban a su provincia para el acontecimiento festivo. Mujeres y hombres con historias diversas marcadas por la experiencia de la migración y las dificultades del desarraigo. En lo personal mi objetivo era conocer que significaba para estas personas volver a su pago, participar en la fiesta. El propósito de conocer implicó sin embargo y de hecho también conocerme, en cuanto detrás de la meta de percibir 
trayectorias de los migrantes emergió con fuerza mi trayectoria familiar y especialmente la recuperación de la historia de mis abuelos migrantes. Muy en particular se trataba de la historia de mi abuela y sus relatos acerca de sus sueños de volver un día a su aldea natal como me narraba cada vez que me contaba algo de su pueblo. En el transcurso del viaje mis apreciaciones comenzaron a cambiar. Era el investigador, pero básicamente era uno más, también yo pasajero en ese transcurrir compartiendo esa vuelta al pago de los otros viajeros. Pero era el investigador atento a lo que podía observar, escuchar, apreciar. Perceptivo de las palabras pero aún más de los gestos y muy especialmente de las miradas. El trayecto a Mailin llevó más de $10 \mathrm{hs}$. pero no solo se trató de compartir el viaje sino que al llegar al pueblo durante los días de la fiesta el micro era nuestro hábitat transitorio, dado que en el lugar no había albergue disponible, lo cual constituía una situación que inevitable nos llevó a compartir con los restantes pasajeros distintos momentos y situaciones. Nuestra participación en la fiesta implicó a su vez momentos de diálogo, de intercambios, de tensiones, como no podía ser de otra manera entre un grupo tan heterogéneo y que en su gran mayoría no se conocía.En cada conversación surgía con fuerza un tipo de creencia y relación personal con el Cristo, nuestro Santito como lo llamaban familiarmente, el sentido profunda de participar en su fiesta.

Las preguntas eran inevitables, pero en realidad las mías eran secundarias ante la necesidad de comprender las preguntas que los mismos participantes se hacían, el desafío de percibir sus respuestas que comenzaban a constituirse en un bagaje de nuevos interrogantes. La búsqueda de sentidos se conformaba más en una apreciación de gestos y comportamientos, de sonrisas y de lágrimas que de conceptos o discursos abstractos. Una situación que ya había comenzado al iniciar el viaje y se había acrecentado al ingresar el micro en la provincia de Santiago y escuchar las expresiones y los vivas emocionados de quienes regresaban a su tierra, aunque sea provisoriamente por unos días. Entre emociones y situaciones aparecían, las palabras cargadas de significado: "siempre vuelvo cuando puedo", "la importancia de estar allî", “ de cumplir su promesa" la expresión repetida acerca de que "es nuestro pago". Así en el marco de un viaje y sus largos recorridos, de las idas y las vueltas por el lugar, del permanecer y mirar y escuchar y compartir ese tiempo de vida se comienza a comprender en el migrante la significación del estar, no como permanecer, sino como experiencia existencial, el sentido de la celebración festiva, la vivencia de su fe. 
Una instancia imprescindible de comprensión superadora de explicaciones pero que abre un camino para la "interacción cognitiva" en la cual el otro hace explícito un relato preñado de sentido (Vasilachis .I, 2013:261). Como nos señalaban algunos migrantes.

Siempre vuelven, por eso vengo a vender para la fiesta porque seguro van a estar ..." (feriante en la fiesta)

todos los años nos encontramos con mis hermanos en Mailin. Siempre viene alguno...Yo voy allí y seguro que alguno está" (entrev. migrante)

Lo que iba registrando con mis sentidos, lo que veía, lo que escuchaba, lo que olía, lo que comía, lo que podía grabar y las múltiples fotografías que podía sacar, todo era una fuente de información que iba conformando mis registros etnográficos Una perspectiva de abordaje en el campo que se constituyó en una fuente de información clave desde donde comprender la significación de ese viaje y de ese "estar allí" para la fiesta. Un abordaje etnográfico constituido más que en un desafío de conocimiento en un aprendizaje hermenéutico.

Finalmente nos interesa destacar la necesidad del reposicionamiento epistemológico que encuentra en la metodología cualitativa y en la etnografía un camino importante a recorrer y una experiencia transformadora a realizar. Una experiencia que aparece como sumamente adecuada cuando se trata de abordar configuraciones de sentido y tramas simbólicas entre las cuales se despliegan creencias, ritualidades y comportamientos de los sujetos creyentes. Un replanteo basado en el reconocimiento de la capacidad cognitiva e interpretativa de los sujetos que les ha sido permanentemente negada por la impronta de un paradigma hegemónico y excluyente. Un posicionamiento sustentado en el convencimiento de que son dichos sujetos los que en primera instancia pueden y deben decir su palabra acompañando lo señalado por Rita. Segato (2010) en cuanto "Solo el margen puede enseñar al margen cómo mejor leer el mundo".

\section{Bibliografía}

Ameigeiras A. (2006) El abordaje etnográfico en la investigación social - pp. 107-152- en I. Vasilachis (coord)- Estrategias de investigación social - GEDISA- Barcelona

Ameigeiras A. (1992) Cultura y religiosidad popular: El entramado socio-cultural de una fiesta santiagueña en el Gran Bs. As - en Stromata Julio Dic. 1992 - no.,-34- 409-417- 
Ameigeiras A. (2002) Fundadores y pasantes: Catolicismo popular y migración en el Gran Bs. As. " pp. 49-61 en A.Ameigeiras-N-.Boulgourdijan-F.Mallimaci-J.Soneira- Religión en tiempos de crisis-Edit. Nobuko- Bs. As-

Ameigeiras A. (2002b) Fiesta popular e identidad religiosa en el Gran Bs. As. En J.Filc(org) Territorios, identidades y fronteras- pp 81-97- EDic. Al Margen- Bs. As.

Algranti J (2008) Cuando lo invisible gobierna lo visible - Etnografía de los cultos de prosperidad en la Iglesia evangélica pentescostal Rey de reyes en "Perspectivas Latinoamericanas, Cuadernos de investigación del mundo latino. Centro de Estudios Latinomaericanos .- Univ. Nanzan , Nagoyá Japón No. 5- Pag-. 37-67

-Barrera Rivera Darío P. (2014) Estruturas e teias de significado: "habitus" e "cultura" nas ciências da religião - en Estudos de Religião, Vol 28 - No. 1.- Univ. Metodista São Paulo-

Becker H. (1996). The epistemology of qualitative research".pp.53-72- en R Jessor, A Colby y RA Shweder (eds) Ethnography and human development. Chicago - The university of Chicago press-

Bidaseca K. (2010). Perturbando el texto colonial - Edit- SB- Bs. As -

Briceño León - R y Sonntag H- (1999). La ciencia social y Amèrica Latina-:la promesa por cumplir- en Briceño León y Sonntag H (eds) El legado de la sociología, la promesa de la ciencias social - Inmanuel -Wallerstein -. Unesco Caracas- pp. 104 - 116- Edit. Nueva Sociedad -Venezuela

Castella Vazquez C (2015) Del desinterés al entusiasmo .El fenómeno religioso en la investigación etnográfica española"- Gaceta de Antropología-31(2)

Castro Gomez, S. Mendietta E. (1998). Introducción; La translocalización discursiva de Latinoamérica en tempos de la globalización en Castro Gomez S Y Mendietta E (ed) Teorías sin disciplina (latinomaericanismo, poscolonialidada y globalización en debate - México -Porrúa (Citado por Bidaseca K - op. cit-.pag. 116)

Denzin N-Y Lincoln (2012) Paradigmas y perspectivas en disputa - Gedisa - Vol II- Argentina-

De la Torre R (2012) Religiosidades nómadas. Creencias y prácticas heterodoxas en GuadalajaraPublicaciones de la Casa Chata - Guadalajara Mexico

Flick Uwe .2004. - Introducción a la investigación cualitativa- Pag. 39- Edic. Morata - Madrid

Guba E, Lincoln Y .1994. Competing Paradigms in Qualitative Research "en Denzin N, K Lincoln Y (eds) "Handbook of Qualitative research"- Thousand oaks California, Sage

Guba E, Lincoln Y.2012.Controversias paradigmáticas, contradicciones y confluencias emergentes - -pag 70 en Denzin N Y Lincoln - Paradigmas y perspectivas en disputa- Edit Gedisa-Barcelona

Kusch R. (1975). América profunda - Edit. Bonum - Bs. As

Kusch R. (1978). Esbozo de una Antropologia Filosófica Americana” Edic. Castañeda - Bs. As.

Lander E. (2000). Ciencias sociales: saberes coloniales y eurocentricos pag.4 en Edgardo Lander (Comp) La colonialidad del saber: eurocentrismo y ciencias sociales. Perspectivas latinoamericanas- Buenos Aires Lugar.CLACSO, 
Lewgoy B. (2004) Etnografía da leitura num grupo de estudos espirita”, pp. 255-281.

Ludueña G. (2006) Etnografía del espacuio en monasterios de clausura en la Argentina”Simbolismo, ritual y performance"- Antropología sociocultural- Bs.As.

-Mareque E. (1989). "Lineas fundamentales del pensamiento de Rodolfo Kusch "53 en E. Azcuy ( comp) Kusch y el pènsar desde América - Edic-. F. Garcia Cambeiro- Bs. As.

Paden W. (1998) religius Worlds. The comparative study of religión. Boston -Beacon Press (Citado por P. Wright. 19898 - pag. 1856

-Parker C. (1993). Otra lógica en América Latina - FCE

Parker C (2011) Cambios religiosos, fronteras míviles e interculturalidad- en En A Higuera Bonfil(coord.) -Religión y cultura contemporáneas. La manda mexico

Rodriguez E (2014) As ciencias siociais sa religiao como ciencias da interpretação, en Estudos de Religião. Vol 28 - No. 1- Univ. Metodista São Paulo.

Sanchis, P.(1998) Estudos de religião; academia e instituições religiosas, um diálogo en construção - p. 50 in Souza B Muñiz de, Gouveia E, Jardilino J, R. Lima (org) Sociología da religião no Brasil. Sao Paulo: PUC/UMESP,

Quijano A. (1992). Colonialidad y modernidad-racionalidad en Bonilla Heraclio (edic) Los conquistadores 1492 y la población indigena de la Américas- Quito Bogota-tercer mundo Flacso-Libri Mundi

Quijano A (2000) Colonialidad del poder, eurocentrismo y América Latina- en Lander E (comp) La colonialidad del saber, eurocentrismo y ciencias sociales. Perspectivas Latinoamericanas.Clacso

Santos, Boaventura de Souza (2009). Una epistemología del sur: La reinvención del conocimiento y la emancipación social - Clacso - Siglo XXI - edit.

Segato R .(2010). Prólogo. p. 12 en Bidaseca K. (2010) Perturbando el texto colonial-Edit SB-Bs. As.

Steil C. (1996) O Sertao das romería. CID-Edit Vozes: Petrópolis.

Vasilachis de Gialdino I. (1992). Métodos cualitativos I - Los problemas teóricos epistemológicos- CEAL - Bs. As.

Varela J, Alvarez Uría F. (1997) Genealogía y sociología. Edic. El cielo por asalto - Bs. As.

Vasilachis de Gialdino I. (2013). Discurso científico, político, jurídico y de resistencia. Edit- Gedisa.

Vasilachis de Gialdino I. (2003). Pobres, pobreza, identidad y representaciones sociales. Edit. GedisaBarcelona

Vasilachis de Gialdino, I. (1997). Discurso politico y prensa escrita. Edit. Gedisa - Barcelona.

Vasilachis de Gialdino I. (2012). De la forma de conocer a las formas de conocer- Prólogo a la edic en castellano pag. 23- en Denzin N Y Lincoln - Paradigmas y perspectivas en disputa- Edit Gedisa-Barcelona. 
Vigil M.J. (2014). Gran transición hacia una nueva visión: Nuevos paradiogmas como retos para la religión en su papel hermeéutico, en Estudos de Religião, Vol. 28 - No. 1.- Univ. Metodista Sao paulo-

Wright Pablo (1998) Etnografía y existencia en la antropología de la religión- 180-193 en Sociedad y religión n. 156-17, Argentina.

Viotti N. Los hombres también llorfan. Masculinidad, sensibilidad y etnografía entre católicos emocionales porteños. Ciencias Sociais e Religião. P. Alegre, Año II, No. 11- pp.35-58,

Wallerstein I .1996. Abrir las ciencias sociales. Madrid: Siglo XXI Edit

Wright P (2008).

Wright P. (1998) Etnografía y existencia en la antropología de la religión. Sociedad y religión No. 16-17, pp. 180-191. 